\title{
Hydrometra: An Uncommon but Important Cause of Cystic Lesion in the Pelvis
}

\author{
Karthik Marpalli Vasudeva ${ }^{1}$, Jini Pullalathu Abraham ${ }^{2}$ \\ ${ }^{1}$ Assistant Professor, Department of Radiodiagnosis, A.J. Institute of Medical Sciences and Research Centre, Mangalore, \\ Karnataka, ${ }^{2}$ Senior Resident, Department of Radiodiagnosis, A.J. Institute of Medical Sciences and Research Centre, \\ Mangalore, Karnataka, India
}

Corresponding author: Karthik Marpalli Vasudeva, Assistant Professor, Department of Radiodiagnosis, A.J. Institute of Medical Sciences and Research Centre, Mangalore, Karnataka, India

DOI: http://dx.doi.org/10.21276/ijcmsr.2020.5.1.33

BY-NC-ND

How to cite this article: Karthik Marpalli Vasudeva, Jini Pullalathu Abraham. Hydrometra: an uncommon but important cause of cystic lesion in the pelvis. International Journal of Contemporary Medicine Surgery and Radiology. 2020;5(1):A153-A154.

\section{A B S T R A C T}

Introduction: Hydrometra is an accumulation of fluid in the endometrial cavity. It can be gross to mimic a cystic lesion in the pelvis. We describe a case of A 63-year-old female patient with hydrometra presenting with a history of postmenopausal bleeding and pain abdomen for 1 week misdiagnosed as an ovarian neoplasm.

Case report: A 63-year-old female patient presented with postmenopausal bleeding and abdominal pain since1 week. A transabdominal ultrasound done elsewhere revealed a large cystic lesion in the pelvis near the right adnexa suspicious for ovarian carcinoma. On per speculum examination a friable growth was seen in both lips of the cervix. A Cervical biopsy was performed, where it was proven to be squamous cell carcinoma. A transvaginal ultrasound and a Staging CT scan revealed a fluid collection in the endometrial cavity with a hypoechoic mass in the cervix. Hydrometra was drained through vaginal route and Radiotherapy was initiated.

Conclusion: Gross hydrometra can mimic an ovarian neoplasm in the Transabdominal ultrasound. A thorough clinical examination and Transvaginal ultrasound will help distinguish the two.

Keywords: Hydrometra, Cervical Carcinoma, Radiotherapy

\section{INTRODUCTION}

Hydrometra is an accumulation of fluid in the endometrial cavity. It can be gross to mimic a cystic lesion in the pelvis. ${ }^{1}$ We describe a case of A 63-year-old female patient with hydrometra presenting with a history of postmenopausal bleeding and pain abdomen for 1 week misdiagnosed as an ovarian neoplasm.

\section{CASE REPORT}

A 63-year-old female patient presented with postmenopausal bleeding and abdominal pain since1 week. She was treated elsewhere for the same complaints, however, symptoms did not subside. She was a known hypertensive on treatment with calcium channel blocker. A transabdominal ultrasound done elsewhere revealed a large cystic lesion in the pelvis near the right adnexa (Figure 1A) suspicious for ovarian carcinoma. On per speculum examination a $4 \times 4 \mathrm{~cm}$ friable growth was seen in both lips of the cervix which bled on touch and was hard inconsistency. On per vaginal examination, a growth was seen involving both lips of cervix extending up to $1 / 3$ rd of the vagina. On per rectal examination, a hard mass was felt anteriorly, the proximal part of the left parametrium was involved, sparing the pelvic sidewall and rectal mucosa was free.

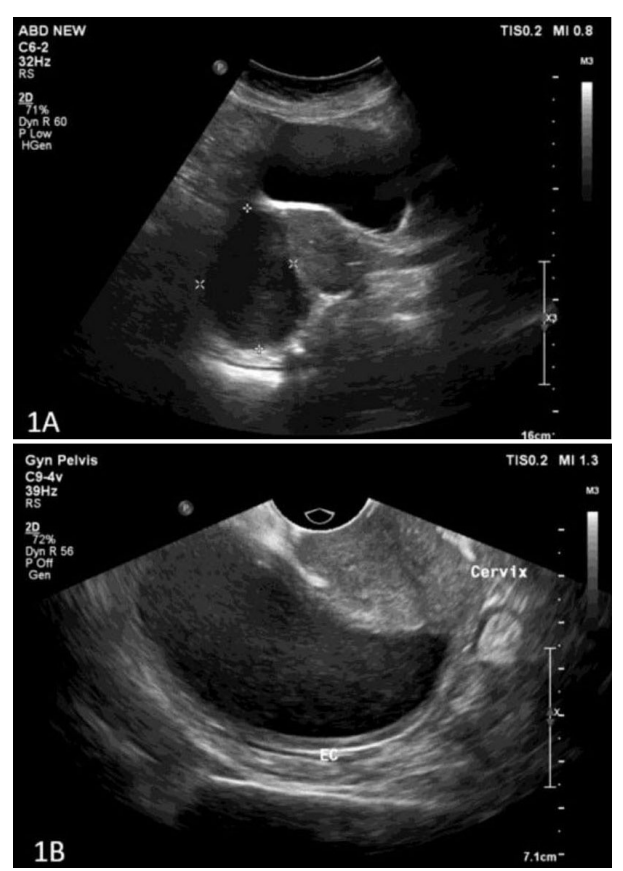

Figure-1: (A) Transabdominal ultrasound- axial view showing a well-defined cyst adjacent to the cervix, (B) Transvaginal ultrasound -sagittal view showing distended endometrial cavity (EC) mimicking a cyst in the pelvis with bulky cervix 


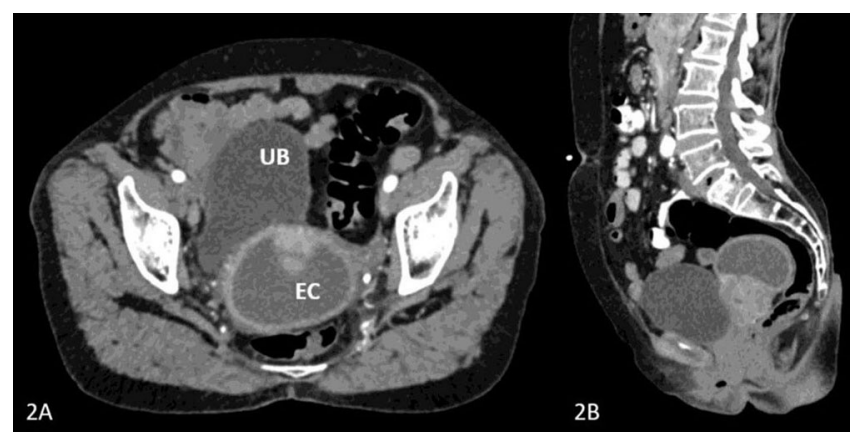

Figure-2: Contrast-enhanced Computed tomography of the abdomen and pelvis-axial (A) sagittal (B) showing hydrometra(EC) posterior to the urinary bladder (UB) with heterogeneously enhancing cervix.

A Cervical biopsy was performed, where it was proven to be squamous cell carcinoma. A transvaginal ultrasound (Figure 1B) revealed a fluid collection in the endometrial cavity with a hypoechoic mass in the cervix. Contrast-enhanced computed tomography (CECT) abdomen and pelvis was performed for Radiotherapy planning (Figure 2A and B), which revealed bulky heterogeneously enhancing cervix and a large fluid collection in the endometrial cavity with retroperitoneal lymphadenopathy.

She underwent cervical dilatation and drainage of the hydrometra which yielded $200 \mathrm{ml}$ of clear fluid. The culture of the fluid was sterile. The patient is currently undergoing radiotherapy for the carcinoma cervix.

\section{DISCUSSION}

Hydrometra is an abnormal fluid collection in the endometrial cavity. Common causes include obstruction of the cervix due to infection, inflammation or malignancies. There are few case reports of hydrometra in cervical carcinoma and endometrial carcinoma. ${ }^{1,2} \mathrm{~A}$ grossly distended uterine cavity may appear cystic. One of the case report mentions about a degenerated fibroid mimicking hydrometra. ${ }^{3}$ It might mimic ovarian lesions due to mass effect and midline shift to one side. Hydrometra can cause symptoms due to mass effect like urethral obstruction.

In our case, the patient reported with postmenopausal bleeding and a transabdominal ultrasound done elsewhere (Figure 1A) reported as suspicious for ovarian carcinoma. On clinical examination and a subsequent transvaginal ultrasound (Figure 1B), this was found to be a distended hydrometra secondary to cervical squamous cell carcinoma. A CECT scan (Figure 2) subsequently showed the ovaries separate from the cystic lesion.

\section{CONCLUSION}

Gross hydrometra can mimic an ovarian neoplasm in a Transabdominal ultrasound. A thorough clinical examination and Transvaginal ultrasound will help distinguish the two.

\section{REFERENCES}

1. Byun JY, Kim SE, Choi BG, Ko GY, Jung SE, Choi KH. Diffuse and focal adenomyosis: MR imaging findings. Radiographics 1999;19(1):S161-70.

2. Takeuchi K, Tsujino T, Sugimoto M, Yoshida S,
Kitazawa S. Endocervical adenocarcinoma associated with lobular endocervical glandular hyperplasia showing rapid reaccumulation of hydrometra. Int $\mathrm{J}$ Gynecol Cancer 2008;18(4):1285-8.

3. Tok Ch, Bux S, Mohamed S, Lim B. Degenerated uterine fibroid mimicking hydrometra: fallacy in CT. Biomed Imaging Interv J 2006;2(5):e42.

4. Tsai, H.-J., Hsu, S.-M., Lee, C.-K. Urethral obstruction caused by a hydrometra. European Journal of Obstetrics \& Gynecology and Reproductive Biology 2014;175(6):211-212.

\section{Source of Support: Nil; Conflict of Interest: None}

Submitted: 05-01-2020; Accepted: 01-02-2020; Published online: 28-02-2020 